\title{
EXTENSIONS OF THE CLASSICAL CESÀRO OPERATOR ON HARDY SPACES
}

\author{
GUILLERMO P. CURBERA and WERNER J. RICKER*
}

\begin{abstract}
For each $1 \leq p<\infty$, the classical Cesàro operator $\mathscr{C}$ from the Hardy space $H^{p}$ to itself has the property that there exist analytic functions $f \notin H^{p}$ with $\mathscr{C}(f) \in H^{p}$. This article deals with the identification and properties of the (Banach) space $\left[\mathscr{C}, H^{p}\right]$ consisting of all analytic functions that $\mathscr{C}$ maps into $H^{p}$. It is shown that $\left[\mathscr{C}, H^{p}\right]$ contains classical Banach spaces of analytic functions $X$, genuinely bigger that $H^{p}$, such that $\mathscr{C}$ has a continuous $H^{p}$-valued extension to $X$. An important feature is that $\left[\mathscr{C}, H^{p}\right]$ is the largest amongst all such spaces $X$.
\end{abstract}

\section{Introduction}

The classical Cesàro operator, given by

$$
\mathscr{C}(f)(z):=\sum_{n=0}^{\infty}\left(\frac{1}{n+1} \sum_{k=0}^{n} a_{k}\right) z^{n},
$$

with $f(z)=\sum_{0}^{\infty} a_{k} z^{k}$ an analytic function on the open unit disc $\mathrm{D}$, is bounded on the Hardy space $H^{p}$, for every $0<p<\infty$. For $1<p<\infty$, this follows from a result of Hardy concerning trigonometric series together with M. Riesz's theorem. The boundedness on $H^{1}$ was proved by Siskakis, who also gave an alternative proof for $1<p<\infty$, [7], [8].

Observe that $\mathscr{C}$ is injective, but not surjective on $H^{p}$ (as 0 belongs to the spectrum of $\mathscr{C},[7])$, that is, $\mathscr{C}$ is not an isomorphism on $H^{p}$. However, $\mathscr{C}$ is an isomorphism on the Fréchet space $H(\mathrm{D})$ of all analytic functions on D. So, there exist analytic functions $f \notin H^{p}$ such that $\mathscr{C}(f) \in H^{p}$. Accordingly, the domain of $\mathscr{C}: H^{p} \rightarrow H^{p}$ is, in a certain natural sense, lacking in size. This raises the question of whether there exist Banach spaces of analytic functions, always meant over D (i.e., vector subspaces of $H(\mathrm{D})$ which are complete for some norm), larger than $H^{p}$ and which $\mathscr{C}$ maps continuously into $H^{p}$ ? If so, does there exist a "largest" such space and what properties would it have?

\footnotetext{
* The authors acknowledge the support of the Maximilian Bickhoff-Universitätsstiftung (Eichstätt, Germany), D.G.I. \#MTM2009-12740-C03-02 (Spain) and FQM 262 (Junta de Andalucía).

Received 25 September 2009.
} 
The first observation is that such a space cannot be $H^{q}$, for any $q \in[1, p)$. Indeed, Aleman and Cima have considered operators $T_{g}$ determined by an analytic symbol $g$ via $T_{g} f(z):=\int_{0}^{z} f(\xi) g^{\prime}(\xi) d \xi$. They have shown, for $1<q<p<\infty$, that $T_{g}$ maps $H^{q}$ into $H^{p}$ if and only if $g$ is in the Lipschitz class $\Lambda_{\alpha}$ with $\alpha:=(1 / q)-(1 / p)$, [1, Theorem 1(iii)]. Since this is not the case for $g(z)=-\log (1-z)$, which corresponds to the Cesàro operator, it follows that $\mathscr{C}$ (taking values in $H^{p}$ ) cannot be extended to any larger $H^{q}$ space as its domain.

In Section 2 we characterize precisely when $\mathscr{C}$ is bounded from a weighted Hardy space $H^{p}(w)$ into $H^{p}$, which allows us to exhibit a class of weights $w$, with certain growth conditions, for which $H^{p} \varsubsetneqq H^{p}(\omega)$ and such that $\mathscr{C}\left(H^{p}(w)\right) \subseteq H^{p}$. Such weights $\omega_{1}$ and $\omega_{2}$ exist for which $H^{p}\left(\omega_{1}\right)$ and $H^{p}\left(\omega_{2}\right)$ are not comparable. In Section 3 we show that there actually does exist a largest Banach space of analytic functions (denoted by $\left[\mathscr{C}, H^{p}\right]$ ) to which $\mathscr{C}$ has a continuous extension and maps into $H^{p}$. In particular, for the above mentioned weights $\omega$ we have that $H^{p}(\omega) \subseteq\left[\mathscr{C}, H^{p}\right]$. This containment is actually proper (as is $H^{p} \subseteq\left[\mathscr{C}, H^{p}\right]$ ). It is precisely this feature, i.e., that $\left[\mathscr{C}, H^{p}\right]$ contains classical Banach spaces of analytic functions which are genuinely larger than $H^{p}$, which makes the space $\left[\mathscr{C}, H^{p}\right]$ interesting. Just as interesting is the optimality of the space $\left[\mathscr{C}, H^{p}\right]$ relative to $\mathscr{C}$, in the sense that it is also the largest Banach space of analytic functions $f$ on $D$ for which the formula (1) produces an element of $H^{p}$ and such that the extended Cesàro operator $\mathscr{C}:\left[\mathscr{C}, H^{p}\right] \rightarrow H^{p}$ is still continuous. Section 3 is also devoted to exposing certain Banach space properties of $\left[\mathscr{C}, H^{p}\right]$, to studying various properties of individual functions from $\left[\mathscr{C}, H^{p}\right]$, which can behave quite differently to those from $H^{p}$, and to identifying the space of all multipliers for $\left[\mathscr{C}, H^{p}\right]$.

\section{Extensions of the Cesàro operator}

A weight is any function $\omega$ on the unit circle T such that $\omega>0$ a.e. and with $\log \omega$ integrable. Let $\psi$ be an outer function corresponding to $\omega$, that is, $\psi$ is analytic on D and $|\psi|=\omega$ a.e. on $\mathrm{T},[5, \S 2.4]$. The weighted Hardy space $H^{p}(\omega)$ associated to $\omega$ is then the Banach space $\psi^{-1 / p} \cdot H^{p}=\{f \in H(\mathrm{D})$ : $\left.\psi^{1 / p} f \in H^{p}\right\}$ with norm $\|f\|_{p, \omega}:=\left\|\psi^{1 / p} f\right\|_{p}$; see, for example, [6].

We state for further reference the following facts.

Proposition 2.1. Let $1 \leq p<\infty$ and $\omega$ be a weight with $\psi$ an outer function corresponding to $\omega$.

(i) Given $\varphi \in H(\mathrm{D})$ the multiplication operator $M_{\varphi}(f):=\varphi \cdot f$ is well defined (and hence, continuous) from $H^{p}$ to $H^{p}$ if and only if $\varphi \in H^{\infty}$.

(ii) $H^{p} \subseteq H^{p}(\omega)$ if and only if $\psi$ is bounded.

(iii) $H^{p}(\omega) \subseteq H^{p}$ if and only if $\psi^{-1}$ is bounded. 
Proof. (i) If $\varphi f \in H^{p}$ for all $f \in H^{p}$, then a closed graph argument shows that $M_{\varphi}$ is continuous. We may assume that $\left\|M_{\varphi}\right\|=1$. Since $\varphi=M_{\varphi}(1) \in$ $H^{p}$, it follows by iteration that $\left\{\varphi^{n}\right\}$ is contained in the closed unit ball of $H^{p}$. Accordingly, $\frac{1}{2 \pi} \int_{0}^{2 \pi}\left|\varphi\left(e^{i \theta}\right)\right|^{n p} d \theta \leq 1$ for all $n \in \mathrm{N}$. By splitting these integrals over the sets $|\varphi|^{-1}([0,1])$ and $|\varphi|^{-1}((1, \infty))$ it follows that $|\varphi| \leq 1$ a.e.

(ii) $H^{p} \subseteq H^{p}(\omega)$ is equivalent to $f \mapsto \psi^{1 / p} f$ being bounded on $H^{p}$ which, by (i), is equivalent to $\psi^{1 / p}$, and hence $\psi$, being bounded.

(iii) $f \in H^{p}(\omega)$ precisely when $f=\psi^{-1 / p} g$ for some $g \in H^{p}$. Hence, $H^{p}(\omega) \subseteq H^{p}$ is equivalent to $g \mapsto \psi^{-1 / p} g$ being bounded on $H^{p}$ which, by (i), is equivalent to $\psi^{-1 / p}$, hence also to $\psi^{-1}$, being bounded.

The following result characterizes those weights $\omega$ with the property that $\mathscr{C}$ maps $H^{p}(\omega)$ continuously into $H^{p}$.

THEOREM 2.2. Let $1 \leq p<\infty$ and $\omega$ be a weight with $\psi$ an outer function corresponding to $w$. The following conditions are equivalent.

(i) $\mathscr{C}: H^{p}(\omega) \rightarrow H^{p}$ continuously.

(ii) The operator which sends $g \in H$ (D) to the function

$$
z \longmapsto \int_{0}^{z} g(\xi) \frac{\psi^{-1 / p}(\xi)}{1-\xi} d \xi, \quad z \in \mathrm{D},
$$

maps $H^{p}$ into itself continuously.

(iii) The function

$$
\rho_{\psi}: z \longmapsto \int_{0}^{z} \frac{\psi^{-1 / p}(\xi)}{1-\xi} d \xi, \quad z \in \mathrm{D},
$$

belongs to the space BMOA.

Proof. Let $f \in H^{p}(\omega)$. Then $f=\psi^{-1 / p}$.g, for some unique $g \in H^{p}$. We require the following well known integral expression for $\mathscr{C}$, namely, for each $h \in H(\mathrm{D})$,

$$
\mathscr{C}(h)(z)=\frac{1}{z} \int_{0}^{z} \frac{h(\xi)}{1-\xi} d \xi, \quad z \in \mathrm{D},
$$

which yields

$$
\mathscr{C}(f)(z)=\frac{1}{z} \int_{0}^{z} g(\xi) \frac{\psi^{-1 / p}(\xi)}{1-\xi} d \xi
$$

Following [2], for analytic functions $\rho$ and $h$ on D, we consider

$$
T_{\rho}(h)(z):=\frac{1}{z} \int_{0}^{z} h(\xi) \rho^{\prime}(\xi) d \xi, \quad z \in \mathrm{D} .
$$


Then $\mathscr{C}(f)=T_{\rho_{\psi}}(g)$ with $\rho_{\psi}$ given by (2). Consequently, $\mathscr{C}$ maps $H^{p}(\omega)$ continuously into $H^{p}$ if and only if $T_{\rho_{\psi}}$ maps $H^{p}$ into itself continuously. Furthermore, [1, Theorem 1(ii)] asserts that this last condition is equivalent to $\rho_{\psi} \in \mathrm{BMOA}$.

Theorem 2.2 makes no assertion concerning any (possible) relationship between $H^{p}(\omega)$ and $H^{p}$. However, combining it with Proposition 2.1 we can deduce, for appropriate $\omega$, that the Cesàro operator has a genuine extension to a larger space $H^{p}(\omega)$ with values still in $H^{p}$.

Corollary 2.3. Let $1 \leq p<\infty$ and $\omega$ be a weight with $\psi$ an outer function corresponding to $w$. Suppose that

(i) $\psi$ is bounded and $\psi^{-1}$ is unbounded, and

(ii) the function $z \mapsto \int_{0}^{z} \frac{\psi^{-1 / p}(\xi)}{1-\xi} d \xi$ belongs to BMOA.

Then $\mathscr{C}: H^{p}(\omega) \rightarrow H^{p}$ continuously and $H^{p} \varsubsetneqq H^{p}(\omega)$.

The following result specifies growth conditions on a weight $\omega$ which are sufficient to ensure that $\mathscr{C}$ maps $H^{p}(\omega)$ continuously into $H^{p}$ and $H^{p} \varsubsetneqq H^{p}(\omega)$.

Corollary 2.4. Let $1 \leq p<\infty$ and $\omega$ be a weight with $\psi$ an outer function corresponding to $w$. Suppose that

(i) $\psi$ is bounded and $\psi^{-1}$ is unbounded, and

(ii) there exist distinct points $a_{1}, \ldots, a_{m} \in \mathrm{T} \backslash\{1\}$ such that

$$
\psi^{-1}(z)=O\left(\frac{1}{\prod_{k=1}^{m}\left|z-a_{k}\right|^{p}}\right), \quad|z| \rightarrow 1^{-} .
$$

Then $\mathscr{C}: H^{p}(\omega) \rightarrow H^{p}$ continuously and $H^{p} \varsubsetneqq H^{p}(\omega)$.

Proof. We could apply condition (iii) of Theorem 2.2. However, we prefer a direct argument which highlights the operator-theoretic approach.

Let $f \in H^{p}(\omega)$. Then $f=\psi^{-1 / p} \cdot g$, for some $g \in H^{p}$. Using (3) and setting $h(z):=\psi^{-1 / p}(z) \prod_{k=1}^{m}\left(z-a_{k}\right)$ yields

$$
\mathscr{C}(f)(z)=\frac{1}{z} \int_{0}^{z} \frac{\psi^{-1 / p}(\xi) g(\xi)}{1-\xi} d \xi=\frac{1}{z} \int_{0}^{z} \frac{h(\xi) g(\xi)}{(1-\xi) \prod_{k=1}^{m}\left(\xi-a_{k}\right)} d \xi .
$$

So, for suitable constants $A_{0}, A_{1}, \ldots, A_{m} \in \mathrm{C}$ (with $a_{0}:=1$ ) we have

$$
\begin{aligned}
\mathscr{C}(f)(z) & =\sum_{k=0}^{m} \frac{A_{k}}{z} \int_{0}^{z} \frac{h(\xi) g(\xi)}{a_{k}-\xi} d \xi=\sum_{k=0}^{m} A_{k} \frac{1}{z} \int_{0}^{z / a_{k}} \frac{h\left(a_{k} \eta\right) g\left(a_{k} \eta\right)}{1-\eta} d \eta \\
& =\sum_{k=0}^{m} \frac{A_{k}}{a_{k}} \mathscr{C}\left(h\left(a_{k} \cdot\right) g\left(a_{k} \cdot\right)\right)\left(z / a_{k}\right) .
\end{aligned}
$$


The function $h$ is, by condition (ii), bounded. Since $z \mapsto z / a_{k}$ and $z \mapsto$ $a_{k} z$ are automorphisms of $\mathrm{D}$, each function $g_{k}(z):=h\left(a_{k} z\right) g\left(a_{k} z\right)$ is in $H^{p}$ and so, $\mathscr{C}\left(g_{k}\right)\left(\cdot / a_{k}\right) \in H^{p}$, for $0 \leq k \leq m$. Consequently, $\mathscr{C}(f) \in H^{p}$. Hence, $\mathscr{C}\left(H^{p}(\omega)\right) \subseteq H^{p}$. This, Lemma 2.5 below, and the fact that point evaluations are continuous linear functionals on both $H^{p}$ and $H^{p}(\omega)$, imply that $\mathscr{C}: H^{p}(\omega) \rightarrow H^{p}$ continuously.

Condition (i) and Proposition 2.1 imply that $H^{p} \varsubsetneqq H^{p}(\omega)$.

If $X$ and $Y$ are Banach spaces of analytic functions, then the vector space containment $X \subseteq Y$ is equivalent to continuity of the inclusion $X \hookrightarrow Y$, provided that point evaluations are continuous on both $X$ and $Y$. Moreover, since point evaluations are continuous on $H(\mathrm{D})$, we always have a continuous inclusion $X \hookrightarrow H(\mathrm{D})$. A similar result holds for the Cesàro operator.

Lemma 2.5. Let $X, Y$ be Banach spaces of analytic functions such that point evaluations are continuous on both $X, Y$. Then $\mathscr{C}(X) \subseteq Y$ if and only if $\mathscr{C}$ maps $X$ into $Y$ continuously.

Proof. We can apply the Closed Graph Theorem. Let $f_{n} \rightarrow 0$ in $X$ and $\mathscr{C}\left(f_{n}\right) \rightarrow g$ in $Y$. By the discussion prior to the lemma, $f_{n} \rightarrow 0$ in $H(\mathrm{D})$. Fix $z \in \mathrm{D} \backslash\{0\}$. Since $f_{n}(\xi) /(1-\xi)$ converges to zero uniformly on the segment $[0, z]$, it follows that $\mathscr{C}\left(f_{n}\right)(z) \rightarrow 0$. But, $\mathscr{C}\left(f_{n}\right)(z) \rightarrow g(z)$. Consequently, $g=0$.

\section{Further extensions of the Cesàro operator}

Can the Cesàro operator be extended beyond the already larger spaces $H^{p}(\omega)$, while still remaining $H^{p}$-valued? Yes, and genuinely. Let us see how to proceed.

As already noted, $\mathscr{C}$ is a topological isomorphism from $H$ (D) onto itself. For $1 \leq p<\infty$, define the linear space

$$
\left[\mathscr{C}, H^{p}\right]:=\left\{f \in H(\mathrm{D}): \mathscr{C}(f) \in H^{p}\right\},
$$

which is then complete with respect to the norm

$$
\|f\|_{\left[\mathscr{C}, H^{p}\right]}:=\|\mathscr{C}(f)\|_{H^{p}} .
$$

Moreover, we have that

$$
H^{p} \subseteq\left[\mathscr{C}, H^{p}\right] \subseteq H(\mathrm{D}) .
$$

The first containment follows from $\mathscr{C}\left(H^{p}\right) \subseteq H^{p}$. Moreover, both inclusions are continuous. This follows from Lemma 2.5 and the fact that point evaluations 
are continuous on $\left[\mathscr{C}, H^{p}\right]$. To see this, fix $z_{0} \in \mathrm{D}$ and let $f \in\left[\mathscr{C}, H^{p}\right]$. Taking into account the identity

$$
g(z)=(1-z)(z \mathscr{C}(g)(z))^{\prime}, \quad g \in H(\mathrm{D}),
$$

which follows from (3), we have, for $\left|z_{0}\right|<r<1$, that

$$
\begin{aligned}
\left|f\left(z_{0}\right)\right| & =\left|1-z_{0}\right| \cdot\left|(z \mathscr{C}(f)(z))^{\prime}\left(z_{0}\right)\right| \\
& =\left|1-z_{0}\right| \cdot\left|\frac{1}{2 \pi i} \int_{|\xi|=r} \frac{\xi \mathscr{C}(f)(\xi)}{\left(\xi-z_{0}\right)^{2}} d \xi\right| \\
& \leq \frac{r^{2}\left|1-z_{0}\right|}{2 \pi\left(r-\left|z_{0}\right|\right)^{2}} \int_{0}^{2 \pi}\left|\mathscr{C}(f)\left(r e^{i \theta}\right)\right| d \theta .
\end{aligned}
$$

Consequently,

$$
\left|f\left(z_{0}\right)\right| \leq \frac{\left|1-z_{0}\right|}{2 \pi\left(1-\left|z_{0}\right|\right)^{2}}\|\mathscr{C}(f)\|_{H^{p}}=\frac{\left|1-z_{0}\right|}{2 \pi\left(1-\left|z_{0}\right|\right)^{2}}\|f\|_{\left[\mathscr{C}, H^{p}\right]} .
$$

Note that the first containment in (6), namely $H^{p} \subseteq\left[\mathscr{C}, H^{p}\right]$, is strict. Indeed, $f(z):=1 /(1+z) \notin H^{1}$ but, $\mathscr{C}(f)(z)=(1 / 2 z) \log ((1+z) /(1-z))$ belongs to every $H^{p}, 1 \leq p<\infty$. Accordingly, $f \in\left[\mathscr{C}, H^{p}\right]$, for all $1 \leq$ $p<\infty$. Clearly, the second containment in (6) is also strict.

Remark 3.1. Fix $1 \leq p<\infty$. Let $X$ be a Banach space of analytic functions. If $\mathscr{C}: X \rightarrow H^{p}$ is continuous, then $\mathscr{C}(X) \subseteq H^{p}$ and so $X \subseteq$ $\left[\mathscr{C}, H^{p}\right]$. On the other hand, suppose that $X \subseteq\left[\mathscr{C}, H^{p}\right]$. Then $\mathscr{C}(X) \subseteq H^{p}$ and hence, if point evaluations are continuous on $X$, it follows from Lemma 2.5 that $\mathscr{C}: X \rightarrow H^{p}$ continuously. This means that $\|\mathscr{C}(f)\|_{H^{p}} \leq M\|f\|_{X}, f \in X$, for some constant $M>0$, that is, $\|f\|_{\left[\mathscr{C}, H^{p}\right]} \leq M\|f\|_{X}, f \in X$. Thus, the natural inclusion $X \subseteq\left[\mathscr{C}, H^{p}\right]$ is necessarily continuous. Consequently, since $\mathscr{C}:\left[\mathscr{C}, H^{p}\right] \rightarrow H^{p}$ is clearly continuous, the space $\left[\mathscr{C}, H^{p}\right]$ can be considered as the "optimal domain" for the operator $\mathscr{C}$, with $\mathscr{C}$ still taking its values in $H^{p}$. That is, $\left[\mathscr{C}, H^{p}\right]$ is the largest of all Banach spaces of analytic functions $X$ such that $\mathscr{C}$ maps $X$ continuously into $H^{p}$. Equivalently, [ $\mathscr{C}, H^{p}$ ] can be interpreted as the largest Banach space of analytic functions to which the Cesàro operator $\mathscr{C}: H^{p} \rightarrow H^{p}$ can be extended, still with all its values in $H^{p}$.

In view of the previous comments, Corollaries 2.3 and 2.4 imply that $H^{p}(\omega) \subseteq\left[\mathscr{C}, H^{p}\right]$ continuously, for all weights $\omega$ satisfying the conditions of these results.

To better understand the nature of individual functions from $\left[\mathscr{C}, H^{p}\right]$ we begin with the following description. 
Proposition 3.2. For each $1 \leq p<\infty$ we have, as vector spaces, that

$$
\left[\mathscr{C}, H^{p}\right]=\left\{f \in H(\mathrm{D}): f(z)=(1-z) g^{\prime}(z) \text { for some } g \in H^{p}\right\} .
$$

Proof. If $f \in\left[\mathscr{C}, H^{p}\right]$, then $\mathscr{C}(f)$ and hence, also $h(z):=z \mathscr{C}(f)(z)$, belongs to $H^{p}$. According to (7) we have $f(z)=(1-z) h^{\prime}(z)$ and so $f$ belongs to the right-hand-side of (9).

Conversely, suppose that $f \in H(\mathrm{D})$ has the form $f(z)=(1-z) g^{\prime}(z)$ for some $g \in H^{p}$. Then

$$
\mathscr{C}(f)(z)=\frac{1}{z} \int_{0}^{z} \frac{(1-\xi) g^{\prime}(\xi)}{1-\xi} d \xi=\frac{g(z)-g(0)}{z}, \quad z \neq 0,
$$

with $\mathscr{C}(f)(0)=g^{\prime}(0)$. Choose $0<\varepsilon<1$ such that $\left|\frac{g(z)-g(0)}{z}-g^{\prime}(0)\right|<\varepsilon$ for $0<|z|<\varepsilon$, in which case $\left|\frac{g(z)-g(0)}{z}\right|<\varepsilon+\left|g^{\prime}(0)\right|$. Moreover, for $\varepsilon \leq|z|<1$ we have $\left|\frac{g(z)-g(0)}{z}\right|<\frac{|g(z)|+|g(0)|}{\varepsilon}$. Hence, $|\mathscr{C}(f)(z)| \leq \alpha|g(z)|+\beta$ for $z \in \mathrm{D}$ and constants $\alpha, \beta>0$. Since $g \in H^{p}$, this implies that $\mathscr{C}(f) \in H^{p}$, that is, $f \in\left[\mathscr{C}, H^{p}\right]$.

Let us deduce some consequences of the previous result. We begin with an alternative function theoretic description of $\left[\mathscr{C}, H^{p}\right]$. By applying to the function $z \mapsto z \mathscr{C}(f)(z)=\int_{0}^{z} \frac{f(\xi)}{1-\xi} d \xi$ (which has value 0 at $z=0$ and whose derivative equals $\left.\frac{f(z)}{1-z}\right)$ the criterion for membership of $H^{p}$ based on the Littlewood-Paley $g$-function, [9, Ch. XIV, Theorems (3.5) and (3.19)], we obtain from Proposition 3.2 the following fact.

Corollary 3.3. Let $1<p<\infty$. Then $f \in\left[\mathscr{C}, H^{p}\right]$ if and only if

$$
\int_{0}^{2 \pi}\left(\int_{0}^{1} \frac{\left|f\left(r e^{i \theta}\right)\right|^{2}}{\left|1-r e^{i \theta}\right|^{2}}(1-r) d r\right)^{p / 2} d \theta<\infty .
$$

Note that, for $p=1$, the above condition is only necessary.

Recall that every element of $H^{p}$, for $1 \leq p<\infty$, has boundary values a.e. on $\mathrm{T}$.

Corollary 3.4. Let $1 \leq p<\infty$. Then there exists a function in $\left[\mathscr{C}, H^{p}\right]$ which fails to have a.e. boundary values. In particular, $H^{p} \varsubsetneqq\left[\mathscr{C}, H^{p}\right]$.

Proof. According to [5, p.92] there exists $g \in H^{\infty} \subseteq H^{p}$ such that $g^{\prime}$ fails to have a.e. boundary values. Then $f(z):=(1-z) g^{\prime}(z)$ belongs to $\left[\mathscr{C}, H^{p}\right]$ (c.f. Proposition 3.2) and $f$ fails to have a.e. boundary values.

REMARK 3.5. (i) The proof of Corollary 3.4 shows there actually exists a function in $\bigcap_{1 \leq p<\infty}\left[\mathscr{C}, H^{p}\right]$ which fails to have a.e. boundary values. 
(ii) Let $\omega$ be a weight as in Section 2 with $\psi$ a corresponding outer function. It is clear from $H^{p}(\omega)=\left\{\psi^{-1 / p} f: f \in H^{p}\right\}$ that every function in $H^{p}(\omega)$ has a.e. boundary values. So, whenever $H^{p}(\omega) \subseteq\left[\mathscr{C}, H^{p}\right]$, Corollary 3.4 implies that the inclusion is proper.

Let Aut(D) denote the group of all automorphisms on D, in which case each space $H^{p}$, for $1 \leq p<\infty$, is invariant under composition with Aut(D). That is, $\left\{f \circ \rho: f \in H^{p}\right\} \subseteq H^{p}$ for all $\rho \in \operatorname{Aut}(\mathrm{D})$.

Proposition 3.6. There exists $\rho \in \operatorname{Aut}(\mathrm{D})$ and $f \in \bigcap_{1 \leq p<\infty}\left[\mathscr{C}, H^{p}\right]$ such that $f \circ \rho \notin\left[\mathscr{C}, H^{1}\right]$. In particular, $f \circ \rho \notin\left[\mathscr{C}, H^{p}\right]$ for $1 \leq p<\infty$.

Proof. The function $f(z):=1 /(1+z)$ satisfies $\mathscr{C}(f)(z)=$ $(2 z)^{-1} \log \left(\frac{1+z}{1-z}\right)$ and so $f \in\left[\mathscr{C}, H^{p}\right]$ for all $1 \leq p<\infty$, that is, $f \in$ $\bigcap_{1 \leq p<\infty}\left[\mathscr{C}, H^{p}\right]$. Of course, $f \notin H^{1}$. Let $\rho(z):=-z$, for $z \in \mathrm{D}$. Then $(f \circ \rho)(z)=1 /(1-z)$ and $\mathscr{C}(f \circ \rho)(z)=1 /(z-1) \notin H^{1}$, that is $f \circ \rho \notin\left[\mathscr{C}, H^{1}\right]$. According to Proposition 3.8(v) below, also $f \circ \rho \notin\left[\mathscr{C}, H^{p}\right]$ for all $1 \leq p<\infty$.

Corollary 3.4 and Proposition 3.6 show that certain "nice" properties of functions from $H^{p}$ fail to be inherited by functions in the larger space [ $\left.\mathscr{C}, H^{p}\right]$. This is not always the case. Proposition 2.1(i) asserts, for $\varphi \in H(\mathrm{D})$, that the operator $M_{\varphi}$ of multiplication by $\varphi$ is defined and continuous from $H^{p}$ into itself precisely when $\varphi \in H^{\infty}$. The same conclusion holds for the spaces $\left[\mathscr{C}, H^{p}\right]$ in place of $H^{p}$.

Theorem 3.7. Let $1 \leq p<\infty$. Given $\varphi \in H(\mathrm{D})$ the multiplication operator $M_{\varphi}(f):=\varphi \cdot f$ is well defined (and hence, continuous) from $\left[\mathscr{C}, H^{p}\right]$ to $\left[\mathscr{C}, H^{p}\right]$ if and only if $\varphi \in H^{\infty}$.

Proof. Suppose first that $\varphi \in H^{\infty}$. Fix $f \in\left[\mathscr{C}, H^{p}\right]$. By Proposition 3.2 there exists $g \in H^{p}$ such that $f(z)=(1-z) g^{\prime}(z)$. Observe that $G \in H(\mathrm{D})$ defined by

$$
G(z):=\varphi(z) g(z)-\int_{0}^{z} \varphi^{\prime}(\xi) g(\xi) d \xi, \quad z \in \mathrm{D}
$$

satisfies

$$
\varphi(z) f(z)=(1-z) \varphi(z) g^{\prime}(z)=(1-z) G^{\prime}(z), \quad z \in \mathrm{D},
$$

and so, again by Proposition 3.2, we see that $\varphi f \in\left[\mathscr{C}, H^{p}\right]$ provided that $G \in$ $H^{p}$. Since $\varphi g \in H^{p}$, to verify that $G \in H^{p}$ it suffices to verify that $T_{\varphi}(g): z \mapsto$ $\int_{0}^{z} \varphi^{\prime}(\xi) g(\xi) d \xi \in H^{p}$. But, $\varphi \in H^{\infty} \subseteq$ BMOA and so indeed $T_{\varphi}(g) \in H^{p}$ for every $g \in H^{p}$, [2, Theorem 1], [1, Theorem 1(ii)]. Accordingly, $\varphi$ has the 
property that $M_{\varphi}(f):=\varphi \cdot f$ belongs to $\left[\mathscr{C}, H^{p}\right]$ whenever $f \in\left[\mathscr{C}, H^{p}\right]$. Using continuity of the point evaluations on $\left[\mathscr{C}, H^{p}\right]$, a closed graph argument shows that $M_{\varphi}:\left[\mathscr{C}, H^{p}\right] \rightarrow\left[\mathscr{C}, H^{p}\right]$ is actually continuous.

Of course, for $p \neq 1$, the above proof can be replaced by a direct appeal to Corollary 3.3.

Conversely, let $\varphi \in H(\mathrm{D})$ be such that $M_{\varphi}:\left[\mathscr{C}, H^{p}\right] \rightarrow\left[\mathscr{C}, H^{p}\right]$ is well defined (and hence, continuous). We may assume that the operator norm of $M_{\varphi}$ satisfies $\left\|M_{\varphi}\right\|=1$. Note, for every $n \geq 1$, that the operator $M_{\varphi^{n}}=\left(M_{\varphi}\right)^{n}$ maps $\left[\mathscr{C}, H^{p}\right]$ into $\left[\mathscr{C}, H^{p}\right]$ and moreover, that $\left\|M_{\varphi^{n}}\right\| \leq 1$. Accordingly, $\varphi^{n} \in\left[\mathscr{C}, H^{p}\right]$ and hence, also $(1-z) \varphi^{n}(z) \in\left[\mathscr{C}, H^{p}\right]$ (because $\left.(1-z) \in H^{\infty}\right)$. Then

$$
\begin{aligned}
\left\|z \mapsto \int_{0}^{z} \varphi^{n}\right\|_{H^{p}} & =\left\|z \mathscr{C}\left((1-z) \varphi^{n}(z)\right)\right\|_{H^{p}}=\left\|\mathscr{C}\left((1-z) \varphi^{n}(z)\right)\right\|_{H^{p}} \\
& =\left\|(1-z) \varphi^{n}(z)\right\|_{\left[\mathscr{C}, H^{p}\right]}=\left\|M_{\varphi^{n}}(1-z)\right\|_{\left[\mathscr{C}, H^{p}\right]} \\
& \leq\left\|M_{\varphi^{n}}\right\| \cdot\|1-z\|_{\left[\mathscr{C}, H^{p}\right]} \leq 1 .
\end{aligned}
$$

From [9, Ch. XIV, Theorem (3.5)] it follows, for some constant $A_{p}>0$ and all $n \geq 1$, that

(10)

$$
\int_{0}^{2 \pi}\left(\int_{0}^{1}\left|\varphi^{n}\left(r e^{i \theta}\right)\right|^{2}(1-r) d r\right)^{p / 2} d \theta \leq A_{p}\left\|z \mapsto \int_{0}^{z} \varphi^{n}\right\|_{H^{p}} \leq A_{p} .
$$

Suppose there exists $z \in \mathrm{D}$ such that $|\varphi(z)|>1$. Then there exists $0 \leq r_{0}<$ $r_{1}<1$ and $0 \leq \theta_{0}<\theta_{1}<2 \pi$ such that $\left|\varphi\left(r e^{i \theta}\right)\right| \geq a$ for some $a>1$ and all $r_{0} \leq r \leq r_{1}$ and $\theta_{0} \leq \theta \leq \theta_{1}$. From (10) we conclude, for all $n \geq 1$, that

$$
\begin{aligned}
A_{p} & \geq \int_{0}^{2 \pi}\left(\int_{0}^{1}\left|\varphi\left(r e^{i \theta}\right)\right|^{2 n}(1-r) d r\right)^{p / 2} d \theta \\
& \geq \int_{\theta_{0}}^{\theta_{1}}\left(\int_{r_{0}}^{r_{1}}\left|\varphi\left(r e^{i \theta}\right)\right|^{2 n}(1-r) d r\right)^{p / 2} d \theta \\
& \geq\left(\theta_{1}-\theta_{0}\right)\left(\left(r_{1}-r_{0}\right) a^{2 n}\left(1-r_{1}\right)\right)^{p / 2} .
\end{aligned}
$$

Since $a>1$, this is impossible. Hence, $|\varphi(z)| \leq 1$ for all $z \in \mathrm{D}$ and so $\varphi \in H^{\infty}$.

Despite the general lack of regularity concerning individual functions from $\left[\mathscr{C}, H^{p}\right]$, the spaces $\left[\mathscr{C}, H^{p}\right]$ exhibit rather good structural properties. Indeed, various Banach space properties of $\left[\mathscr{C}, H^{p}\right]$ follow directly from the fact that $\mathscr{C}$ maps $\left[\mathscr{C}, H^{p}\right]$ linearly and isometrically onto $H^{p}$, for $1 \leq p<\infty$; see (5). Some immediate consequences are as follows. 
Proposition 3.8. Let $1 \leq p<\infty$ and $\left[\mathscr{C}, H^{p}\right]$ be the optimal domain for the Cesàro operator $\mathscr{C}$ on $H^{p}$.

(i) $\left[\mathscr{C}, H^{p}\right]$ is separable.

(ii) $\left[\mathscr{C}, H^{p}\right]$ is uniformly convex (in particular, reflexive) for $p \neq 1$.

(iii) For $p=2,\left[\mathscr{C}, H^{2}\right]$ is a Hilbert space. In particular,

$$
f(z)=\sum_{0}^{\infty} a_{n} z^{n} \in\left[\mathscr{C}, H^{2}\right] \Longleftrightarrow\left(\frac{1}{n+1} \sum_{0}^{n} a_{k}\right) \in \ell^{2} .
$$

(iv) Polynomials are dense in $\left[\mathscr{C}, H^{p}\right]$.

(v) $\left[\mathscr{C}, H^{p_{2}}\right] \varsubsetneqq\left[\mathscr{C}, H^{p_{1}}\right]$ whenever $1 \leq p_{1}<p_{2}<\infty$.

Proof. The isometry between $\left[\mathscr{C}, H^{p}\right]$ and $H^{p}$ immediately yields (i), (ii) and (v). For (iii), observe that $f \in\left[\mathscr{C}, H^{2}\right]$ if and only if $\mathscr{C}(f) \in H^{2}$ if and only if $\left(\frac{1}{n+1} \sum_{0}^{n} a_{k}\right) \in \ell^{2}$; see (1). Finally, for (iv), let $f \in\left[\mathscr{C}, H^{p}\right]$ and $\varepsilon>0$. Since $\mathscr{C}(f) \in H^{p}$, choose $N$ and $\left(b_{k}\right)_{0}^{N} \subseteq \mathrm{C}$ so that $\left\|\mathscr{C}(f)-\sum_{0}^{N} b_{k} z^{k}\right\|_{H^{p}}<\varepsilon$. Taking into account that $\mathscr{C}\left(z^{k}-z^{k+1}\right)=z^{k} /(k+1)$ for $k \geq 0$, we can write

$$
\begin{aligned}
\left\|\mathscr{C}(f)-\sum_{0}^{N} b_{k} z^{k}\right\|_{H^{p}} & =\left\|\mathscr{C}(f)-\sum_{0}^{N} b_{k}(k+1) \mathscr{C}\left(z^{k}-z^{k+1}\right)\right\|_{H^{p}} \\
& =\left\|\mathscr{C}\left(f-\sum_{0}^{N} b_{k}(k+1)\left(z^{k}-z^{k+1}\right)\right)\right\|_{H^{p}} \\
& =\left\|f-\sum_{0}^{N} b_{k}(k+1)\left(z^{k}-z^{k+1}\right)\right\|_{\left[\mathscr{C}, H^{p}\right]} .
\end{aligned}
$$

REMARK 3.9. Concerning $p=\infty$, the definition given in (4) still makes sense and generates the space $\left[\mathscr{C}, H^{\infty}\right]$ for which (5) is again a complete norm. Since $\left[\mathscr{C}, H^{\infty}\right] \varsubsetneqq\left[\mathscr{C}, H^{p}\right]$ continuously, for all $1 \leq p<\infty$ (see (5)), it follows from (8) that point evaluations are continuous on $\left[\mathscr{C}, H^{\infty}\right]$ and

$$
\left|f\left(z_{0}\right)\right| \leq \frac{\left|1-z_{0}\right|}{2 \pi\left(1-\left|z_{0}\right|\right)^{2}}\|f\|_{\left[\mathscr{C}, H^{\infty}\right]} .
$$

However, since $\mathscr{C}$ is not continuous on $H^{\infty}$, we do not have the inclusion $H^{\infty} \subseteq\left[\mathscr{C}, H^{\infty}\right]$ corresponding to (6) for $p=\infty$.

Optimal domains exhibit good behaviour with respect to interpolation via the Petree $K$-method; see [3, Ch.5.\$1]. 
Proposition 3.10. Let $1<p<\infty$ and $\left[\mathscr{C}, H^{p}\right]$ be the optimal domain for the Cesàro operator $\mathscr{C}$ on $H^{p}$. Then

$$
\left(\left[\mathscr{C}, H^{1}\right],\left[\mathscr{C}, H^{\infty}\right]\right)_{1-\frac{1}{p}, p}=\left[\mathscr{C}, H^{p}\right] .
$$

Proof. Note that $\left[\mathscr{C}, H^{\infty}\right] \subseteq\left[\mathscr{C}, H^{1}\right]$ since $H^{\infty} \subseteq H^{1}$. Fix $f \in\left[\mathscr{C}, H^{1}\right]$. Let $f=g_{1}+g_{2}$ with $g_{1} \in\left[\mathscr{C}, H^{1}\right]$ and $g_{2} \in\left[\mathscr{C}, H^{\infty}\right]$. This is equivalent to $\mathscr{C}(f)=\mathscr{C}\left(g_{1}\right)+\mathscr{C}\left(g_{2}\right)$ with $\mathscr{C}\left(g_{1}\right) \in H^{1}$ and $\mathscr{C}\left(g_{2}\right) \in H^{\infty}$ which, in turn, is equivalent to $\mathscr{C}(f)=h_{1}+h_{2}$ with $h_{1} \in H^{1}$ and $h_{2} \in H^{\infty}$ (since $\mathscr{C}$ is an isomorphism between $\left[\mathscr{C}, H^{p}\right]$ and $\left.H^{p}, 1 \leq p \leq \infty\right)$. The isometry between $\left[\mathscr{C}, H^{p}\right]$ and $H^{p}$ then gives

$$
K\left(f, t ;\left[\mathscr{C}, H^{1}\right],\left[\mathscr{C}, H^{\infty}\right]\right)=K\left(\mathscr{C}(f), t ; H^{1}, H^{\infty}\right), \quad t>0 .
$$

Remark 3.11. Following the procedure given in Remark 3.9 for defining the space $\left[\mathscr{C}, H^{\infty}\right]$, we can also consider the Banach space $[\mathscr{C}, \mathrm{BMOA}]$ consisting of those functions $h \in H(\mathrm{D})$ such that $\mathscr{C}(h) \in \mathrm{BMOA}$. We have $H^{\infty} \subseteq[\mathscr{C}, \mathrm{BMOA}]$ and BMOA $\varsubsetneqq[\mathscr{C}, \mathrm{BMOA}]$; see [4, Section 3].

The space $[\mathscr{C}, \mathrm{BMOA}]$ arises naturally as the space $M\left(H^{p},\left[\mathscr{C}, H^{p}\right]\right)$ of functions generating continuous multiplication operators from $H^{p}$ into [ $\left.\mathscr{C}, H^{p}\right]$. Indeed, $\varphi \in M\left(H^{p},\left[\mathscr{C}, H^{p}\right]\right)$ means precisely that $\varphi f \in\left[\mathscr{C}, H^{p}\right]$ for every $f \in H^{p}$, that is, $\mathscr{C}(\varphi f) \in H^{p}$ for every $f \in H^{p}$. Hence, we have the bounded operator (mapping into $H^{p}$ ) given by

$$
f \mapsto \mathscr{C}(\varphi f): z \mapsto \frac{1}{z} \int_{0}^{z} f(\xi) \frac{\varphi(\xi)}{1-\xi} d \xi, \quad f \in H^{p} .
$$

It follows from [2, Theorem 1] that the function $z \mapsto \int_{0}^{z} \frac{\varphi(\xi)}{1-\xi} d \xi$ belongs to BMOA. Consequently, $\mathscr{C}(\varphi) \in \mathrm{BMOA}$, showing that

$$
M\left(H^{p},\left[\mathscr{C}, H^{p}\right]\right)=[\mathscr{C}, \mathrm{BMOA}] .
$$

The optimal domain space $\left[\mathscr{C}, H^{p}\right]$ of the Cesàro operator (for $1 \leq p<\infty$ ) has been identified not just as a linear space properly containing $H^{p}$, but also as a Banach space of analytic functions in its own right possessing various properties. Moreover, for certain weights $\omega$, the weighted Hardy space $H^{p}(\omega)$ is properly and continuously included in $\left[\mathscr{C}, H^{p}\right]$. It would be interesting to find further examples of classical Banach spaces of analytic functions $X$ such that $H^{p} \varsubsetneqq X \varsubsetneqq\left[\mathscr{C}, H^{p}\right]$ continuously.

ACKNOWLEDGEMENT. We thank the referee for some valuable suggestions. 


\section{REFERENCES}

1. Aleman, A., and Cima, J. A., An integral operator on $H^{p}$ and Hardy's inequality, J. Anal. Math. 85 (2001), 157-176.

2. Aleman, A., and Siskakis, A. G., An integral operator on $H^{p}$, Complex Variables Theory Appl. 28 (1995), 149-158.

3. Bennett, C., and Sharpley, R., Interpolation of Operators, Pure Appl. Math. 129, Academic Press, Boston 1988.

4. Danikas, N., and Siskakis, A. G., The Cesàro operator on bounded analytic functions, Analysis 13 (1993), 295-299.

5. Duren, P. L., Theory of $H^{p}$ Spaces, Pure Appl. Math. 38, Academic Press, New York-London 1970.

6. Kisliakov, S., and Xu, Q., Partial retractions for weighted Hardy spaces, Studia Math. 138 (2000), 251-264.

7. Siskakis, A. G., Composition semigroups and the Cesàro operator on $H^{p}$, J. London Math. Soc. (2) 36 (1987), 153-164.

8. Siskakis, A. G., The Cesàro operator is bounded on $H^{1}$, Proc. Amer. Math. Soc. 110 (1990), 461-462.

9. Zygmund, A., Trigomometric Series, Cambridge Univ. Press, Cambridge 1977.

FACULTAD DE MATEMÁTICAS

UNIVERSIDAD DE SEVILLA

APTDO. 1160

SEVILLA 41080

SPAIN

E-mail: curbera@us.es
MATH.-GEOGR. FAKULTÄT

KATHOLISCHE UNIVERSITÄT EICHSTÄTT-INGOLSTADT

D-85072 EICHSTÄTT

GERMANY

E-mail: werner.ricker@ku-eichstaett.de 